\title{
All the Little Pieces \\ - Regulation of Mitochondrial Fusion and Fission by Ubiquitin and Small Ubiquitin-Like Modifier and Their Potential Relevance in the Heart -
}

\author{
Makhosazane Zungu, PhD; Jonathan Schisler, PhD; Monte S. Willis, MD, PhD
}

\begin{abstract}
Mitochondria are dynamic organelles that undergo a constant cycle of division and fusion to maintain their function. The process of mitochondrial fusion has the effect of mixing their content, allowing complementation of protein components, mtDNA repair, and distribution of metabolic intermediates. Fission, on the other hand, enables mitochondria to increase in number and capacity, and to segregate mitochondria for autophagy by the lysosome ("mitophagy"). Disruption of these protein quality control mechanisms has recently been identified in multiple cardiac diseases, including cardiac hypertrophy, heart failure, dilated cardiomyopathy, and ischemic heart disease, and is intimately tied to mitochondrial control of apoptosis. Proteins that regulate mitochondrial fusion and fission have been discovered, including Mfn1, Mfn2, and Opa1 (fusion) and Drp1 and Fis1 (fission). In this review, we discuss how these proteins are regulated by post-translational modification with ubiquitin and SUMO (small ubiquitin-like modifier). We then present what is known about the ubiquitin and SUMO ligases that regulate these post-translational modifications and regulation of mitochondrial fusion and fission, exploring their potential as therapeutic targets of cardiac disease. (Circ J 2011; 75: 2513-2521)
\end{abstract}

Key Words: Heart; Mitochondrial dynamics; SUMO; Ubiquitin

$\mathbf{M}$ itochondria are highly dynamic cellular organelles involved in a wide array of physiological functions, including apoptosis, ATP production, calcium and iron homeostasis, lipid metabolism, aging, and the production and attenuation of reactive oxygen species (ROS). Although these organelles are generally thought to be morphologically static, they actually alter their morphology continuously in response to various cellular cues, which is a phenomenon termed "mitochondrial dynamics". These alterations involve mitochondrial division (fission) and the merging of individual mitochondria (fusion).

Mitochondria are double-membrane structures that comprise an inner and outer mitochondrial membrane separated by an inter-membrane space. Contact sites between the inner and outer mitochondrial membranes consist of components of the mitochondrial permeability transition pore (PTP) and serve as sites for fission and fusion. ${ }^{1}$ There is growing evidence that the proteins that mediate mitochondrial fusion and fission are regulated by post-translational modifications, including ubiquitination and SUMOylation. These novel mechanisms of regulation involve complex networks of enzymes that specifically place ubiquitin or SUMO (small ubiquitin-like modifier), respec- tively, on key proteins that regulate fission and fusion (eg, Fis1, Drp1, Mfn1/2, and Opa1). In this review, we summarize the known ubiquitin proteasome system and SUMOylation pathways that regulate fission and fusion, and discuss the relevance of these processes in the context of cardiac mitochondrial bioenergetics and disease.

\section{Mitochondrial Fission and Fusion}

Mammalian mitochondria are mobile organelles that undergo repeated cycles of fission and fusion. Mitochondrial fission and fusion events require GTP hydrolysis and are crucial for normal mitochondrial function, as well as for the redistribution of lipids and proteins throughout the cell. ${ }^{2}$ In the context of mitochondrial physiology, fission is necessary for the correct redistribution of mitochondrial DNA during cell division ${ }^{3}$ and for transporting mitochondria to daughter cells during mitosis and meiosis. ${ }^{4}$ In contrast, fusion is a mechanism by which neighboring mitochondrial membranes fuse. This process occurs as a means of recovering the activities of damaged/ depolarized membranes, which thereby ensures the proper mixing of metabolites and mitochondrial DNA (mtDNA).,5

Received September 5, 2011; accepted September 29, 2011; released online October 15, 2011

Department of Pathology \& Laboratory Medicine (M.Z., M.S.W.), Department of Internal Medicine, Division of Cardiology (J.S.) and McAllister Heart Institute (M.S.W.), University of North Carolina, Chapel Hill, NC, USA

Mailing address: Monte S. Willis, MD, PhD, McAllister Heart Institute, Department of Pathology \& Laboratory Medicine, University of North Carolina, Medical Biomolecular Research Building, Rm 2336, 103 Mason Farm Road, Chapel Hill, NC 27599-7525, USA. E-mail: monte_willis@med.unc.edu

ISSN-1346-9843 doi:10.1253/circj.CJ-11-0967

All rights are reserved to the Japanese Circulation Society. For permissions, please e-mail: cj@j-circ.or.jp 
Another view is that mitochondrial fusion allows for genetic complementation between 2 mitochondria, which promotes ATP synthesis in oxygen-deprived regions of a cell through electrical transmission of mitochondrial membrane potential across the mitochondrial network. The energy derived from GTP hydrolysis during fusion is essential to facilitate the transmission of calcium signals and membrane potential across distances in the cell.6,7 Mitochondrial biogenesis is highly compartmentalized in nerve and myocytes and is adversely affected by mutations in proteins involved in mitochondrial fusion and fission. ${ }^{89}$ Twig et al. demonstrated that certain mitochondria within a single cell can spontaneously lose their membrane potential, while the majority of the organelles maintain it, suggesting a possible mechanism of compartmentalizing metabolic defects that allows for aberrant mitochondria to be targeted for proteasomal degradation. ${ }^{5}$

Maintaining mitochondrial morphology directly correlates with the metabolic stability of the cell. ${ }^{10-12}$ Disruption of mitochondrial fission results in a large network of fused mitochondria, whereas the disruption of fusion yields small and dividing mitochondria. Aberrant mitochondrial fission results in impaired mitochondrial recruitment and localization, ${ }^{7,13}$ suggesting that fission events are crucial for mitochondrial trafficking within cells. Impaired mitochondrial fusion results in decreased membrane potential, decreased oxygen consumption, and decreased mtDNA replication. ${ }^{10}$ In cardiomyocytes, perturbations in mitochondrial fission and fusion can lead to apoptosis, which is a prominent mechanism of cardiomyocyte loss in heart failure.

\section{Regulators of Mitochondrial Fusion: Mfn1, Mfn2, and Opa1}

Mitochondrial fusion events are mediated by GTPase dynamin-family proteins, including mitofusin 1 (Mfn1), mitofusin 2 (Mfn2), and optic atrophy-1 (Opa1) (Figure 1). ${ }^{10,14}$ Mfn1 and Mfn2 localize to the outer mitochondrial membrane and promote fusion. Opa1 is found in the inner mitochondrial membrane, facing the inter-mitochondrial space, and interacts with Mfn1 during mitochondrial fusion. ${ }^{14}$ Mfn1 and Mfn2 share approximately $77 \%$ sequence similarity; replacement with either Mfn1 or Mfn2 to Mfn-null cells rescues their function, indicating their redundant activities. ${ }^{15} \mathrm{Mfn} 1$ and Mfn2 expression has been identified in rat and mouse hearts by reverse transcription polymerase chain reaction. ${ }^{16,17}$ In the absence of Mfn1, Mfn2, or Opa1, mitochondrial fission is enhanced because of the inhibited mitochondrial fusion that occurs. ${ }^{15,18}$ Despite these functional overlaps, Mfn1 and Mfn2 play distinct roles in mitochondrial fusion. Mfn 1 has higher affinity for GTPase and facilitates the initial GTP-dependent outer mitochondrial membrane tethering. ${ }^{19}$ In contrast, Mfn2 exhibits weak GTPase activity and GTP-dependent oligomerization and is mainly involved in the mixing of juxtaposed outer mitochondrial membrane and in facilitating docking of the inner mitochondrial membrane. ${ }^{19}$

Studies have shown that Opa1 maintains the cristae structure by tethering, but not fusing, the inner mitochondrial membrane at cristae necks, and therefore maintains the folded structure. $^{18,20,21}$ The mechanisms that control Opa1-mediated inner mitochondrial membrane tethering have not been delineated. However, it was recently shown that Opa1-mediated inner mitochondrial fusion may occur secondary to a signal from active mitofusins on the outer mitochondrial membrane. ${ }^{22}$ Opa1 expression levels are decreased in depolarized mitochondria within intact cells. ${ }^{23}$ This observation was confirmed by studies demonstrating that Opa1 cleavage is increased when the electrochemical potential across the inner mitochondrial membrane is decreased, suggesting that Opa1 may regulate the bioenergetic state and mitochondrial morphology..$^{20,24,25}$ The inhibition of fusion augments the membrane potential, ${ }^{10,18}$ suggesting that diminished fusion capacity could either be the cause or consequence of mitochondrial membrane depolarization. The downregulation of Opa1 expression by RNAi results in spontaneous apoptosis, which suggests that Opa1-mediated mitochondrial fusion additionally protects cells from apoptosis. ${ }^{18}$

\section{Regulators of Mitochondrial Fission: Drp1, Fis1, and, Mff}

Mitochondrial fission can be divided into several phases: (1) initial constriction of mitochondrial tubules; (2) mobilization of cytosolic dynamic-related protein 1 (Drp1); (3) recruitment of Drp1 to the mitochondria; (4) assembly of a scission complex; and (5) the actual scission event; followed by disassembly of the scission complex ${ }^{26,27}$ (Figure 1). Although the mechanism for the initial constriction of the membrane is not known, the final constriction and scission process is mediated by Drp1-driven GTP binding and hydrolysis. It has been postulated that the mitochondrial fission factor (Mff) may recruit proteins that mediate the initial constriction or could act in combination with the human fission 1 (Fis1) protein to form the scission complex. ${ }^{28}$

\section{Drp1 and Fis1}

DLP1 (dynamin-like protein 1)/Drp1 (called Drp1 from hereon) transcript levels are abundantly expressed in skeletal muscle, heart, kidney, and brain of adult humans. ${ }^{29}$ Drp1 is primarily found in the cytosol, localizing to foci in the outer mitochondrial membrane where future fission sites occur during fission. ${ }^{30}$ Drp1 lacks a mitochondrial targeting sequence and is recruited by Fis1 to the outer mitochondrial membrane to mediate fission. ${ }^{28,30-32}$ Translocation of Drp1 is dependent on actin and microtubules, because the disruption of F-actin and the retrograde motor protein complex dynein/dynactin impairs the translocation of Fis1 and therefore inhibits fission events. ${ }^{33}$ Drp1-deficient cells have impaired mitochondrial oxidative capacity, suggesting that Drp1 may play a crucial role in the maintenance of mitochondrial ATP production. ${ }^{34}$ Drp1 is mainly localized to the cytosol, and to a lesser extent at mitochondrial tips, where it oligomerizes around the mitochondria and promotes their fission in a GTP-dependent manner. ${ }^{30,35}$

The Fis 1 protein is a $17-\mathrm{kDa}$ outer mitochondrial protein that promotes the fission of peroxisomes and mitochondria, mainly by serving as a receptor for Drp1. Fis1 is abundantly expressed in isolated mitochondria from many tissues, including the heart. ${ }^{36}$ The structure of Fis1 resembles that of proteins involved in mitochondrial import. ${ }^{37}$ Moreover, the overexpression of Fis 1 in cells promotes mitochondrial fission and results in Drp1-dependent cytochrome c release and apoptosis. ${ }^{38}$

\section{Regulation of Mitochondrial Fusion and Fission Events by the Ubiquitin Proteasome System (UPS) and SUMOylation}

The UPS and SUMOylation pathway are multi-faceted coordinated systems found in the heart (Figure 2 for details). The specificity of both systems comes from the E3 proteins, termed the ubiquitin and SUMO ligases, respectively. Here we highlight recent discoveries that uncovered the identity of multiple 


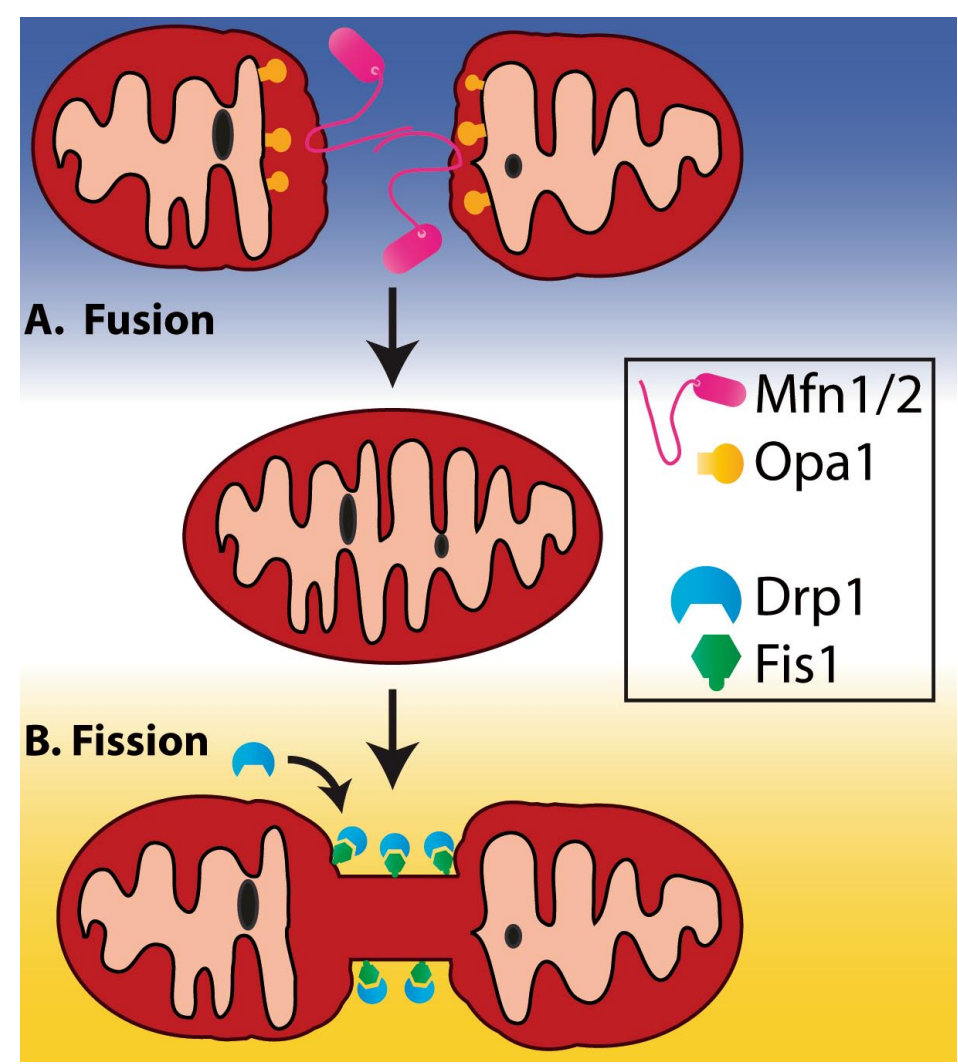

Figure 1. Mitochondrial fusion and fission. Mitochondria are dynamic organelles that continually change their morphology between elongated interconnected networks and fragmented disconnected units. Enhanced fission and fusion have been associated with metabolic stress, autophagy, and apoptosis and implicated in cardiac disease (see text). (A) The proteins mitofusin 1 and mitofusin 2 (Mfn1/2) and the optic atrophy-1 (Opa1) proteins play key roles in regulating mitochondrial fusion. The mitofusin isoforms Mfn1/2 (in pink) are found in the external mitochondrial membrane with their GTPase domain oriented to the cytosol. Mfn1 and Mfn2 interact with each another to coordinate the fusion of the external membrane of opposing mitochondria. ${ }^{15,69}$ Opa1 (in yellow) is found in the intermembrane space and associated with the internal mitochondrial membrane. It participates in remodeling of the fusion of the internal membrane and remodeling of the mitochondrial cristae. ${ }^{25,70}$ (B) The key components of mitochondrial fission include the activity of dynamin-related protein-1 (Drp-1), which is found in the cytoplasm (indicated in blue) localized to points of the external mitochondrial membrane (future fission sites), and fission protein 1 (Fis1), found in the outer mitochondrial membrane (in green). Drp1 shares homology with dynamins, which are GTPases that regulate vesicle trafficking and endocytosis. ${ }^{71}$ Lacking a mitochondrial target sequence, Drp1 is recruited to mitochondria by the adaptor protein Fis $1 .{ }^{71}$

E3 proteins from the ubiquitin and SUMO systems, which regulate fusion and fission proteins, and therefore mitochondrial dynamics. Specifically, we review the ubiquitin ligase MARCH5 (membrane-associated RING finger (C3HC4) 5), the SUMO protease SENP5, and the dual ubiquitin and SUMO ligase, MULAN/MAPL (mitochondrial ubiquitin ligase activator of $\mathrm{NF}-\kappa \mathrm{B} /$ mitochondrial-anchored protein ligase) (summarized in Table 1). Although they are all present in the heart, most of the data presented here demonstrates their regulation of mitochondria dynamics at the molecular level in non-cardiac systems. The extrapolation of these E3 protein activities into the heart is an exciting new frontier of investigation, with implications discussed in the following section.

\section{Mitochondrial MARCH5}

The mitochondrial ubiquitin-ligase, MARCH5 protein (also known as MITOL [mitochondrial ubiquitin ligase]), is an integral outer mitochondrial membrane protein found in the human heart. ${ }^{39}$ Not only has MARCH5 been implicated in mitochondrial protein quality control in disease processes, ${ }^{40-42}$ recent studies have implicated MARCH5 in regulating mitochondrial fission and fusion proteins in the heart. Because fission and fusion are intimately involved with mitochondrial protein quality control, MARCH5 may broadly, therefore, be involved in multiple aspects of mitochondrial protein quality control.

MARCH5 regulates the turnover of super oxide dismutase 1 (SOD1) and misfolded proteins involved in PolyQ-mediated cytotoxicity found in common neurodegenerative diseases, including Alzheimer and Huntington diseases. ${ }^{43,44}$ Using mutant SOD1 (mSOD1) as a model of misfolded proteins, it has been shown that MARCH5 interacts with mSOD1, but not wildtype SOD1, to ubiquitinate the protein. ${ }^{43}$ Decreasing MARCH5 by siRNA results in an accumulation of mSOD1 in mitochondria, enhancing mSOD1-induced generation of ROS and cell death. These studies indicate the MARCH5 plays a role in removing mSOD1 by ubiquitin-dependent degradation, resulting in cellular protection. ${ }^{43} \mathrm{MARCH} 5$ also interacts with and ubiquitinates misfolded proteins that underlie polyglutamine (commonly called PolyQ)-based diseases in the brain. To 
A

\section{Ubiquitination}

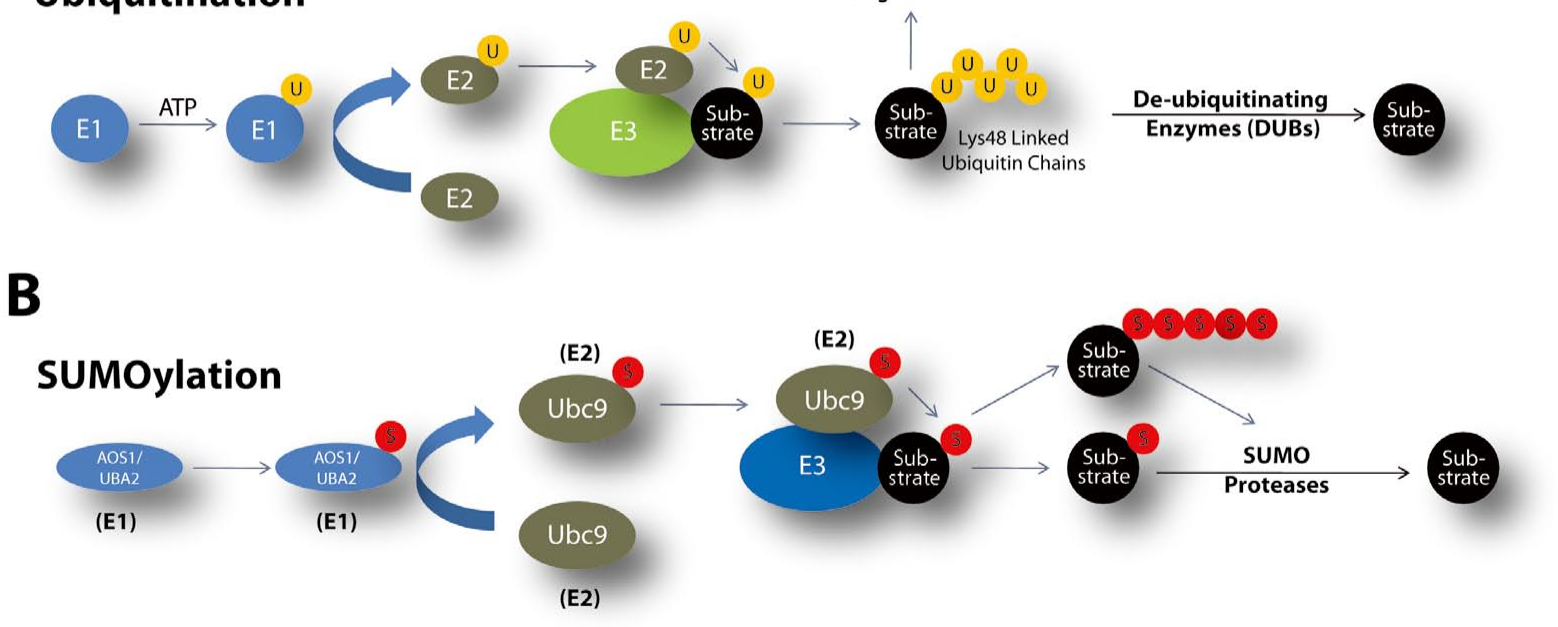

Figure 2. Mitochondrial proteins are selectively regulated by post-translational modifications with ubiquitin or SUMO. (A) The ubiquitin proteasome system (UPS). Post-translational modification of specific substrates with ubiquitin occurs by the cooperative interaction of multiple enzymes. Initially, the E1 (ubiquitin-activating enzyme) catalyzes ubiquitin C-terminal acyl-adenylation and creates a thioester bond and an AMP leaving group. This is followed by the transfer of ubiquitin by a ubiquitin conjugating enzyme (E2), which also forms a thioester bond between its cysteine and ubiquitin. Finally, ubiquitin is transferred to a lysine residue of the substrate (shown in black) by a ubiquitin ligase (E3). The progressive transfer of additional activated ubiquitin proteins to the Lys48 of a previously conjugated ubiquitin moiety results in polyubiquitination. The polyubiquitin chain serves as a direct recognition site for the $26 \mathrm{~S}$ proteasome or can be bound to a shuttling protein and transported to the proteasome. Following the conjugation of ubiquitin to the target protein by a ubiquitin ligase, the target protein is degraded by the proteasome and free ubiquitin is recycled. 72 (B) The SUMOylation pathway. Post-translational modifications of proteins by small ubiquitin-like modifier (SUMO) occur by mechanisms that parallel ubiquitination. Initially, free SUMO is cleaved by SUMO-1/sentrin-specific protease (SENP) and activated by the formation of a thioester bond between the exposed C-terminal glycine and the catalytic cysteine of the E1 activating enzyme. ${ }^{73}$ This reaction is dependent on ATP hydrolysis. SUMO is then transferred to the catalytic cysteine of a single E2 conjugating enzyme (Ubc9). The interaction between the E2 and E3 allows E3 to initiate the transfer of SUMO from E2 to the specific substrate that the SUMO E3 interacts with directly. ${ }^{74}$ The counterbalance of substrate SUMOylation by SUMO ligases are the SUMO isopeptidases (SENP, aka proteases), which counterbalance the SUMO E3s by cleaving off SUMO from specific substrates. ${ }^{75}$ SUMOylation may involve the addition of a single SUMO moiety (mono-SUMOylation), multiple SUMO moieties (polySUMOylation), or the addition of heterogeneous moieties consisting of SUMO and ubiquitin. Unlike SUMO-1, SUMO-2 and SUMO3 have the ability to form isopeptide bonds between the C-terminal glycine residue of a SUMO molecule and Lys 11 of another molecule of SUMO-2/-3, which leads to poly-SUMOylation. ${ }^{76}$ Therefore, SUMO-2/-3 targets may be more susceptible to ubiquitination and subsequent degradation. The placement of SUMO on specific substrates has been shown to regulate diverse biological processes within the nucleus, nuclear pores, and cytosol to affect protein localization, activity, stabilization, and interaction with other proteins. ${ }^{77,78}$ SUMO-1 is predominantly conjugated to substrates and exists in limited amounts, whereas SUMO-2, and its almost identical isoform SUMO-3, exist in abundant amounts in vivo as free form monomers. ${ }^{79}$

determine this, studies were performed using a model of PolyQ disease involving the expression of a highly toxic, misfolded $\mathrm{N}$-terminal truncated ataxin-3. A link between the accumulation of pathogenic ataxin-3 ( $\triangle \mathrm{NAT}-3 \mathrm{Q} 7)$ and cell death/disease progression has been established experimentally. ${ }^{45}$ Recent studies identified that MARCH5 is protective against $\triangle \mathrm{NAT}$ 3Q7 cellular toxicity by its ability to ubiquitinate and degrade this mutant protein, again demonstrating its role as a ubiquitin ligase in mitochondrial protein quality control. ${ }^{44}$

Given MARCH5's regulation of mitochondrial protein quality at the molecular level, it is not too surprising that MARCH5 also regulates mitochondrial fusion and fission, another form of protein quality control. MARCH5 regulates mitochondrial fusion and fission by ubiquitinating Mfn2, Fis1, and Drp1.41,42 This was discovered by increasing MARCH5 levels in COS7 cells. ${ }^{46}$ Increased MARCH5 was colocalized to the mitochondrial membrane, and caused mitochondrial elongation in approximately $40 \%$ of transfected cells. ${ }^{46}$ However, mitochondrial elongation was prevented when the Flag-tagged MARCH5 was co-expressed with an Mfn2 mutant lacking the transmembrane span (Mfn2 $\Delta \mathrm{TM}) .{ }^{46}$ Most of these cells (70\%) co-transfected with MARCH5 and the mutant Mfn2 not only did not elongate, they promoted mitochondrial fission. These studies indicate how MARCH5 enhances fusion, in part, by its ability to ubiquitinate Mfn2. These studies and others suggest that MARCH5 alone lacks the intrinsic ability to fuse mitochondria and its regulation of fusion may be mediated largely through Mfn2 activity. ${ }^{41,46}$

However, MARCH5's ability to regulate mitochondrial fission is more complex than its interaction with and targeted degradation of the fusion protein Mfn2 just described. MARCH5 also interacts with, ubiquitinates, and degrades the fission proteins Fis1 and Drp1. HeLa cells expressing either a MARCH5 lacking the ubiquitin ligase domain or treated with siRNA 


\begin{tabular}{|c|c|c|c|c|}
\hline & Substrate(s) & How regulated? & Consequence & References \\
\hline \multicolumn{5}{|l|}{ Ubiquitin ligases } \\
\hline \multirow{5}{*}{$\begin{array}{l}\text { MARCH5 (membrane-associated } \\
\text { RING finger (C3HC4) 5) }\end{array}$} & Mutant SOD1 & Ubiquitination & Reduced mSOD-induced mitochondrial toxicity & 41 \\
\hline & $\begin{array}{l}\text { N-terminal-truncated } \\
\text { ataxin-3 (Poly-Q } \\
\text { disease mutation) }\end{array}$ & Ubiquitination & $\begin{array}{l}\text { Regulates mitochondrial localization of } \triangle \text { NAT- } \\
\text { 3Q37, Attenuates } \triangle \text { NAT-3Q37-mediated cellular } \\
\text { toxicity }\end{array}$ & 42 \\
\hline & Fis1 & Ubiquitination & Degradation (overall MARCH5 enhances fission) ${ }^{*}$ & 39,40 \\
\hline & Drp1 & Ubiquitination & $\begin{array}{l}\text { Changes subcellular trafficking (overall MARCH5 } \\
\text { enhances fission) }\end{array}$ & 39,40 \\
\hline & Mfn2 & Ubiquitination & Degradation (overall MARCH5 enhances fission) ${ }^{\star}$ & 40,42 \\
\hline $\begin{array}{l}\text { Mitochondrial ubiquitin ligase activator } \\
\text { of NF- } \kappa \text { B (MULAN aka MAPL) }\end{array}$ & Unknown & ND & $\begin{array}{l}\text { Enhances mitochondrial fission (when MULAN } \\
\text { expression increased) }\end{array}$ & 45 \\
\hline \multicolumn{5}{|c|}{ SUMO ligases and proteases (isopeptidases) } \\
\hline $\begin{array}{l}\text { Mitochondrial-anchored protein ligase } \\
\text { (MAPL aka MULAN) }\end{array}$ & Drp1 & $\begin{array}{l}\text { Increased activity } \\
\text { and stability of } \\
\text { Drp1 }\end{array}$ & Enhances mitochondrial fission & 47,46 \\
\hline $\begin{array}{l}\text { SENP5 (SUMO-1/sentrin-specific } \\
\text { peptidase 5) }\end{array}$ & Drp1 & SUMOylated & Enhances mitochondrial fission & 48,49 \\
\hline
\end{tabular}

*MARCH5 ubiquitinates both fusion and fission proteins; the overall effects do not necessarily reflect the individual effects of ubiquitination (described in more detail in text).

SUMO, small ubiquitin-like modifier; SOD, superoxide dismutase; Fis1, fission protein 1; Drp1, dynamic-related protein 1; Mfn, mitofusin; NF, nuclear factor; ND, not determined.

MARCH5 to knock-down MARCH5 expression resulted in an aberrant mitochondrial morphology (ie, enhanced fission). ${ }^{42,47}$ Adding a dominant negative Drp1 to these cells blocked this enhanced fission, implicating Drp1 as a target of MARCH5. ${ }^{42}$ These same studies identified that not only was Drp1 a target of MARCH5, but they also identified that both Drp1 and the fission protein Fis1 were ubiquitinated in the presence of MARCH5, which enhanced their turnover in pulse-chase experiments. ${ }^{42}$ The functional result of MARCH5's regulation of Fis1 was seen experimentally in these studies. Overexpression of Fis1 induced mitochondrial fragmentation; however, the overexpression phenotype of Fis 1 was reverted by co-overexpression of MARCH5, suggesting that its ubiquitination of Fis1 had functional consequences (ie, inhibiting Fis1-regulated mitochondrial fission). ${ }^{42}$ This is consistent with the MARCH5 overexpression studies referenced earlier, which promoted the formation of long tubular mitochondria. ${ }^{41} \mathrm{Im}$ munoprecipitation studies demonstrated that MARCH5 interacts with ubiquitinated Drp1 and promotes Drp1 ubiquitination. ${ }^{41}$ Therefore, MARCH5 regulates the fission phenotype by ubiquitinating fusion proteins (eg, Mfn2) as well as fission proteins (eg, Drp1 and Fis1). MARCH5 ubiquitinates Drp1 and Fis $1,{ }^{41,42}$ but does not affect the stability of Drp1. ${ }^{40}$ Therefore, the ubiquitination of Drp1 by MARCH5 may regulate the subcellular trafficking of Drp1, influencing the rate of mitochondrial fission. ${ }^{40}$

\section{Mitochondrial MULAN/MAPL}

The mitochondrial ubiquitin-ligase, MULAN/MAPL (mitochondrial-anchored protein ligase), is another mitochondrial membrane protein involved in mitochondrial dynamics and mitochondrial-nuclear trafficking. MULAN/MAPL is a $40-\mathrm{kDa}$ protein with orthologs in flies, vertebrates, and plants and consists of 2 transmembrane domains that mediate its localization to the mitochondrial outer membrane. ${ }^{47}$ MULAN is oriented such that its E3 active C-terminal RING finger is exposed to the cytosol, where it has access to other components of the ubiquitin and SUMO systems in the cytosol.

In HeLa cells ectopically expressing MULAN, fragmenta- tion of the long, tubular-shaped mitochondria found in the control cells was recently reported. ${ }^{47,48}$ There was a high level of mitochondrial fragmentation, along with collapse of the mitochondrial network, in such a way that little discernable tubular structure remained. ${ }^{47,48}$ MULAN appeared by immunofluorescence to localize in the perinuclear region. These mitochondrial phenotypes were dose-dependent and were observed in additional cell lines, including HEK293, NIH3T3, COS7, and the cardiac-derived cell lines H9C2 (rat) and HL-1 (mouse) ${ }^{47}$ Knocking down MULAN also perturbed mitochondrial dynamics, although it was not as evident as that seen in the cells ectopically expressing MULAN. When MULAN expression was decreased, the tubular-shaped mitochondria remained visible; increases in fragmentation relative to controls were not as marked ${ }^{47}$ Although the MULAN expressed in these studies was capable of ubiquitinating substrates, as evidenced by auto-ubiquitination, the substrates affecting mitochondrial fusion and/or fission were not studied. Therefore, it remains to be determined which of the proteins regulating fusion and/or fission is targeted by MULAN.

MULAN/MAPL also has SUMO ligase activity that appears to regulate mitochondrial dynamics. Recent studies have identified that the SUMO ligase, MULAN/MAPL, regulates mitochondrial fission, by acting upon and stabilizing Drp $1{ }^{49}$ Increasing MULAN/MAPL leads to an increase in mitochondrial fragmentation in HeLa cells; when MULAN/MAPL does not contain its ligase RING finger domain, this mitochondrial fragmentation does not occur. ${ }^{48}$ Recent studies have shown that MULAN/MAPL has SUMO ligase activity, which is able to place SUMO on Drp1 ${ }^{49}$ To further elucidate how MULAN/ MAPL regulates the fission protein Drp1, siRNA MULAN/ MAPL studies were undertaken. Silencing MULAN/MAPL $(\sim 58 \%)$ in stable HeLa cells led to a nearly $50 \%$ decrease in the levels of endogenous SUMO-1 conjugates, while leaving the total levels of ubiquitinated proteins unchanged ${ }^{49}$ Transient siRNA MULAN/MAPL in HeLa cells silenced MULAN/ MAPL (58\%), leading to a $24 \%$ decrease in SUMO conjugates. ${ }^{49}$ Silencing MULAN/MAPL lead to a $17 \%$ decrease in Drp1 levels; Drp1 itself was found to have less SUMOylation 
(reduced 70\%). ${ }^{49}$ In vitro studies demonstrated that MULAN/ MAPL could SUMOylate endogenously derived Drp1 with SUMO-1. ${ }^{49}$ Immunoprecipitation studies also determined that MAPL interacts with Drp1 specifically. ${ }^{49}$ These studies demonstrate that increasing MULAN/MAPL stimulates fission by SUMOylating Drp1 and enhancing its activity. ${ }^{49}$ However, these studies found that MULAN/MAPL-induced decreases in mitochondria fusion also occur independent of the regulation of fission (ie, Drp1); proteins regulating fusion may also be affected. The details of this mechanism remain to be delineated and reported.

\section{Mitochondrial SENP5}

The SUMO protease (isopeptidase) SENP5 (SUMO-1/sentrinspecific peptidase 5) has recently been shown to regulate mitochondrial fission through its interaction with the fission protein Drp1, in a manner similar to MAPL. This relationship was first identified when Drp1 was identified as an interacting partner with and substrate for SUMO-1. ${ }^{50}$ SUMO-1 was often at the site of mitochondrial fission and remained tightly associated with the tips of mitochondrial fragments. ${ }^{50}$ Subsequent studies identified that SENP5 was the SUMO protease involved in the regulation of mitochondrial dynamics. ${ }^{51}$ Increasing SENP5 expression rescued SUMO-1 induced mitochondrial fragmentation; silencing of SENP5 resulted in enhanced mitochondrial fragmentation and altered morphology. ${ }^{51}$ Drp1 was monoSUMOylated in those studies, suggesting that increased Drp1 SUMOylation enhanced Drp1-mediated fission. ${ }^{51}$ Subsequent studies found that silencing SENP5 leads to cell cycle arrest at a time when SENP5 is translocated to the mitochondria, suggesting a role of SENP5-mediated mitochondrial fission in mitosis. ${ }^{52}$

\section{Regulation of Mitochondrial Dynamics Regulators in Cardiac Hypertrophy and Heart Failure}

Although myocytes demonstrate variations in mitochondrial size and shape, little is known about their dynamics in the differentiated cardiomyocyte and in disease. Mitochondria in cardiomyocytes are highly organized and are situated between the contractile apparatus (sarcomere) next to the $\mathrm{T}$ tubules directly under the sarcolemma..$^{53}$

\section{Drp1 in Cardiomyocytes}

Changes in fusion and fission lead to cardiomyocyte-specific defects experimentally. For example, when neonatal ventricular myocytes express a dominant negative form of Drp1, it results in the inhibition of fission, and leads to many cellular changes. ${ }^{54}$ Attenuating Drp1 prevents the overproduction of ROS, the formation of the mitochondrial PTP, and decreased cell death. ${ }^{54}$

\section{Cardiac Hypertrophy}

Recent studies have identified that mitochondrial fusion and fission proteins are targets of the $\mathrm{Na}^{+} / \mathrm{H}^{+}$exchanger 1 (NHE-1) in cardiomyocyte hypertrophy induced in vitro. When isolated adult rat cardiomyocytes were stimulated by the $\alpha$-agonist, phenylephrine, the fusion protein Mfn2 was inhibited and the fission proteins Drp1 and Fis1 were enhanced. ${ }^{55}$ NHE-1 was implicated in this process because it inhibits the changes in fusion and fission proteins induced in cardiomyocyte hypertrophy using the model described above. This study links for the first time NHE-1, the inhibition of fusion protein expression, and enhanced fission protein expression in the development of cardiac hypertrophy.

\section{Heart Failure}

Cardiac hypertrophy commonly involves a transition to heart failure, during which time numerous changes take place in the myocytes and extracellular matrix (see recent review ${ }^{56}$ ). Recent studies have identified that increased $\mathrm{Ca}^{2+}$ can lead to a rapid and transient mitochondrial fragmentation of cardiomyocytes, in a manner dependent on Drp1 and which enhances ROS generation. ${ }^{53}$ As $\mathrm{Ca}^{2+}$ overload is a feature of heart failure, increases in mitochondrial fission and dysfunction may occur, leading to further injury. ${ }^{57}$ Dividing mitochondria have been seen in failing hearts compared with controls, which has been associated with decreases in the fusion protein, Opa1, and increases in apoptosis in post-myocardial infarction-induced heart failure. ${ }^{58}$ Disorganization and reduced cristae density have been reported in rats with failing hearts. ${ }^{59}$ Although the mechanisms by which mitochondrial dynamics are regulated in cardiomyocytes have not been elucidated in heart failure, dysregulation of both fusion (Mfn1/2, Opa1) and/or fission (Drp1, Fis1) is emerging as an important contributor. ${ }^{57}$

\section{Dilated Cardiomyopathy (DCM)}

DCM is characterized as heart failure with systolic dysfunction and dilated ventricles, although the time course and severity of the disease varies. It is often associated with sudden death and has been associated with a number of hereditary mutations (see recent review ${ }^{60}$ ). The end stage of DCM has been associated with abnormally small and dividing mitochondria, as has hibernating myocardium, and congenital heart diseases. ${ }^{61-64}$ Recent studies have found that mutations in fission regulatory genes can be an underlying cause of DCM. A large-scale mutagenesis study recently identified that a mutation in Drp1 leads to abnormal mitochondria and peroxisomes. ${ }^{65}$ Mice heterozygous for this mutation have reduced levels of mitochondrial enzyme complexes and suffer from cardiac ATP depletion. ${ }^{65}$ Taken together, these studies demonstrate that DCM is associated with enhanced fragmentation of mitochondria, and that dominant mutations in fission genes (ie, Drpl) can lead to a DCM phenotype.

\section{Ischemia/Reperfusion (I/R) Injury}

Cardiomyocytes deal with the oxidative stress induced by $\mathrm{I} / \mathrm{R}$ injury using numerous systems (see recent review ${ }^{66}$ ). Recent studies have identified that alterations in mitochondrial morphology affect the susceptibility of the heart to I/R injury. In these studies, a cardiac-derived cell line (HL-1) was challenged with simulated I/R and monitored for mitochondrial morphology. They identified that HL-1 mitochondrial fission was augmented in response to I/R challenge in a manner that was dependent on the fusion proteins Mfn1, Mfn2, and the fission protein Drp1. ${ }^{67}$ Transfection of HL-1 cells with Mfn1 or Mfn2 or a dominant negative form of Drp1 increased the number of cells with elongated mitochondria after I/R challenge. ${ }^{67}$ Enhancing fusion or decreasing fission thus decreased the mitochondrial PTP and reduced cell death after I/R injury. ${ }^{67}$ Treating HL-1 cells with the mitochondrial division inhibitor-1, a pharmacological inhibitor of Drp1, similarly attenuated the I/R damage to HL-1 cells. ${ }^{67}$ In vivo treatment with this mitochondrial division inhibitor-1 similarly reduced myocardial infarct size in mice subject to coronary artery occlusion and reperfusion. ${ }^{67}$ These studies implicate the regulation of mitochondrial fission in $\mathrm{I} / \mathrm{R}$ cardioprotection; detailed molecular mechanisms by which this mitochondrial fission in $\mathrm{I} / \mathrm{R}$ is regulated remain to be determined. It is not clear if the phenotype represents inhibited fission or enhanced fusion that leads to these benefits. 


\begin{tabular}{|c|c|c|c|c|}
\hline Cardiac disease & Model & Effects of fusion/fission & Mechanism (process affected) & References \\
\hline \multirow[t]{2}{*}{$\mathrm{HF}$} & Post-MI induced HF & $\begin{array}{l}\text { Enhanced mitochondrial } \\
\text { fragmentation }\end{array}$ & $\begin{array}{l}\text { Decreased Opa1 (fusion)/increased } \\
\text { apoptosis }\end{array}$ & 56 \\
\hline & Dilated cardiomyopathy & $\begin{array}{l}\text { Abnormal mitochondria and } \\
\text { peroxisomes }\end{array}$ & Drp1 (fission) & 63 \\
\hline Post-infarction & $\begin{array}{l}\text { Permanent coronary ligation } \\
\text { Sprague-Dawley rats }\end{array}$ & $\begin{array}{l}\text { Enhanced mitochondrial } \\
\text { fission }\end{array}$ & $\begin{array}{l}\text { Decreased Mfn2 and Opa1 } \\
\text { (fusion), Increased Fis1 (fission) }\end{array}$ & 53 \\
\hline
\end{tabular}

MI, myocardial infarction; HF, heart failure; Opa1, optic atrophy-1. Other abbreviations see in Table 1.

Recent studies have also implicated cardiac microRNA (miR) in the regulation of mitochondrial dynamics by regulating Drp1 and mitochondrial fission. Specifically, increasing miR-499, which is abundant in the normal heart, has been shown to prevent I/R-induced apoptosis by regulating the calcineurin-mediated activation of Drp1 (and subsequent mitochondrial fission). ${ }^{68}$ Downstream apoptosis is also inhibited, possibly through miR-499 regulated changes in mitochondrial dynamics. These studies identified that cardiac-specific constitutive overexpression of miR-499 in transgenic mice decreased I/R-induced cardiac hypertrophy and collagen deposition compared with wild type controls. ${ }^{68}$ In I/R, they also found that miR-499 may be regulated by p53 transcription, to inhibit apoptosis through miR-499 and its regulation of mitochondrial fission. The studies indicate a novel mechanism by which cardiac miRs regulate fission through Drp1, complementing ways in which the UPS and SUMO systems post-translationally regulate key proteins involved in mitochondrial dynamics.

Post-infarction, mitochondrial fusion and fission proteins are also regulated. Recent studies have reported that permanent coronary artery ligation in Sprague-Dawley rats results in the regulation of fusion and fission proteins at 12 and 18 weeks after the insult. In whole heart lysates, the fission protein Fis 1 is increased by $80 \%$ and $31 \%$ after 12 and 18 weeks, respectively. ${ }^{55}$ The fusion protein Mfn2 was reduced by $17 \%$ and $22 \%$, in parallel, and Opa1 was decreased by $18 \%$ at 18 weeks (no change at 12 weeks)..$^{55}$ These studies demonstrate that post-myocardial infarction, fusion proteins are inhibited, while fission proteins are increased, paralleling the heart's response to cardiomyocyte hypertrophy, DCM, and I/R injury. The mechanisms that regulate these changes and the changes in the mitochondria themselves have yet to be delineated in more detail in future studies.

These studies indicate a role of mitochondrial fusion and fission in common cardiac diseases by the regulation of specific proteins, including Mfn1/2, Opa1, and Drp1. How these proteins are regulated in the heart post-transcriptionally has not yet been determined, but their regulation does occur in cardiomyocytes experimentally. Increasing evidence implicates both ubiquitin and SUMO modification in the regulation of these proteins, as discussed in the previous section. Because all 3 of the recently described ubiquitin and SUMO ligases (MARCH5, MULAN/MAPL, and SENP5) are found in the heart, it is quite possible they are integral to the regulation of mitochondrial fusion and fission in cardiac disease. However, the role of cardiac ubiquitin and SUMO ligases in the heart has yet to be tested directly. Given the growing role of the UPS in cardiac disease, it would not be surprising if the ubiquitin and SUMO ligases described here, as well as yet to be described ligases, play a significant role of cardiac health and disease. This would be consistent with the broader role of the UPS and SUMO system in regulating protein quality control. There are clear implications of harnessing these protein quality control systems in future therapies for common cardiac diseases.

\section{Summary}

Mitochondria are dynamic organelles that undergo a constant cycle of division and fusion to maintain their function. The process of mitochondrial fusion has the effect of mixing their content, allowing complementation of protein components, mtDNA repair, and distribution of metabolic intermediates. Fission, on the other hand, enables mitochondria to increase in number and capacity, and to segregate mitochondria for autophagy by the lysosome ("mitophagy"). Disruption of these protein quality control mechanisms has recently been identified in cardiac diseases, including cardiac hypertrophy, heart failure, DCM, and ischemic heart disease, and is intimately tied to mitochondrial control of apoptosis (Table 2). Fusion is regulated by the proteins Mfn1, Mfn2, and Opa1, while fission is regulated by the proteins Drp1 and Fis1 (Figure 1). These proteins and others have been described in both cardiac and non-cardiomyocyte cells and are regulated by novel post-translational modifications (Figure 2) mediated by specific ubiquitin and SUMO ligases (Table 1). Although the therapeutic potential of targeting mitochondrial ubiquitin and SUMO ligases with the intention of improving their function has not yet been tested, it may prove to be a valuable and specific mechanism currently unexplored in cardiac disease.

\section{Acknowledgment}

The authors' laboratory is supported by the National Heart, Lung, and Blood Institute grant R01HL104129 (to M.S.W.).

\section{Disclosures}

None.

\section{References}

1. Reichert AS, Neupert W. Mitochondriomics or what makes us breathe. Trends Genet 2004; 20: 555-562.

2. Nakada K, Inoue K, Hayashi J. Interaction theory of mammalian mitochondria. Biochem Biophys Res Commun 2001; 288: 743-746.

3. Ong SB, Hausenloy DJ. Mitochondrial morphology and cardiovascular disease. Cardiovasc Res 2010; 88: 16-29.

4. Hales KG. The machinery of mitochondrial fusion, division, and distribution, and emerging connections to apoptosis. Mitochondrion 2004; 4: 285-308.

5. Twig G, Hyde B, Shirihai OS. Mitochondrial fusion, fission and autophagy as a quality control axis: The bioenergetic view. Biochim Biophys Acta 2008; 1777: 1092-1097.

6. Skulachev VP. Mitochondrial filaments and clusters as intracellular power-transmitting cables. Trends Biochem Sci 2001; 26: 23-29.

7. Szabadkai G, Simoni AM, Chami M, Wieckowski MR, Youle RJ, Rizzuto R. Drp-1-dependent division of the mitochondrial network blocks intraorganellar $\mathrm{Ca}^{2+}$ waves and protects against $\mathrm{Ca}^{2+}$-mediated apoptosis. Mol Cell 2004; 16: 59-68.

8. Li Z, Okamoto K, Hayashi Y, Sheng M. The importance of dendritic mitochondria in the morphogenesis and plasticity of spines and synapses. Cell 2004; 119: 873-887. 
9. Verstreken P, Ly CV, Venken KJ, Koh TW, Zhou Y, Bellen HJ. Synaptic mitochondria are critical for mobilization of reserve pool vesicles at Drosophila neuromuscular junctions. Neuron 2005; 47: $365-378$.

10. Chen $\mathrm{H}$, Chan DC. Emerging functions of mammalian mitochondrial fusion and fission. Hum Mol Genet 2005; 14(Spec No.2): R283R289.

11. Yu T, Robotham JL, Yoon Y. Increased production of reactive oxygen species in hyperglycemic conditions requires dynamic change of mitochondrial morphology. Proc Natl Acad Sci USA 2006; 103: $2653-2658$

12. Pich S, Bach D, Briones P, Liesa M, Camps M, Testar X, et al. The Charcot-Marie-Tooth type 2A gene product, Mfn2, up-regulates fuel oxidation through expression of OXPHOS system. Hum Mol Genet 2005; 14: 1405-1415.

13. Frieden M, James D, Castelbou C, Danckaert A, Martinou JC, Demaurex N. $\mathrm{Ca}(2+)$ homeostasis during mitochondrial fragmentation and perinuclear clustering induced by hFis1. J Biol Chem 2004; 279: 22704-22714.

14. Cipolat S, Martins de Brito O, Dal Zilio B, Scorrano L. OPA1 requires mitofusin 1 to promote mitochondrial fusion. Proc Natl Acad Sci USA 2004; 101: 15927-15932.

15. Chen H, Detmer SA, Ewald AJ, Griffin EE, Fraser SE, Chan DC. Mitofusins Mfn1 and Mfn2 coordinately regulate mitochondrial fusion and are essential for embryonic development. J Cell Biol 2003; 160: $189-200$

16. Rojo M, Legros F, Chateau D, Lombes A. Membrane topology and mitochondrial targeting of mitofusins, ubiquitous mammalian homologs of the transmembrane GTPase Fzo. J Cell Sci 2002; 115: $1663-$ 1674.

17. Bach D, Pich S, Soriano FX, Vega N, Baumgartner B, Oriola J, et al. Mitofusin-2 determines mitochondrial network architecture and mitochondrial metabolism: A novel regulatory mechanism altered in obesity. J Biol Chem 2003; 278: 17190-17197.

18. Olichon A, Baricault L, Gas N, Guillou E, Valette A, Belenguer P, et al. Loss of OPA1 perturbates the mitochondrial inner membrane structure and integrity, leading to cytochrome c release and apoptosis. J Biol Chem 2003; 278: 7743-7746.

19. Ishihara N, Eura Y, Mihara K. Mitofusin 1 and 2 play distinct roles in mitochondrial fusion reactions via GTPase activity. J Cell Sci 2004; 117: $6535-6546$

20. Griparic L, van der Wel NN, Orozco IJ, Peters PJ, van der Bliek AM. Loss of the intermembrane space protein Mgm1/OPA1 induces swelling and localized constrictions along the lengths of mitochondria. J Biol Chem 2004; 279: $18792-18798$.

21. Wong ED, Wagner JA, Gorsich SW, McCaffery JM, Shaw JM, Nunnari J. The dynamin-related GTPase, Mgm1p, is an intermembrane space protein required for maintenance of fusion competent mitochondria. J Cell Biol 2000; 151: 341-352.

22. Hoppins S, Lackner L, Nunnari J. The machines that divide and fuse mitochondria. Аппи Rev Biochem 2007; 76: 751-780.

23. Chen H, Chomyn A, Chan DC. Disruption of fusion results in mitochondrial heterogeneity and dysfunction. J Biol Chem 2005; 280: 26185-26192.

24. Ishihara N, Fujita Y, Oka T, Mihara K. Regulation of mitochondrial morphology through proteolytic cleavage of OPA1. EMBO J 2006; 25: 2966-2977.

25. Meeusen S, DeVay R, Block J, Cassidy-Stone A, Wayson S, McCaffery $\mathrm{JM}$, et al. Mitochondrial inner-membrane fusion and crista maintenance requires the dynamin-related GTPase Mgm1. Cell 2006; 127: 383-395.

26. Labrousse AM, Zappaterra MD, Rube DA, van der Bliek AM. C. elegans dynamin-related protein DRP-1 controls severing of the mitochondrial outer membrane. Mol Cell 1999; 4: 815-826.

27. Frank S, Gaume B, Bergmann-Leitner ES, Leitner WW, Robert EG, Catez F, et al. The role of dynamin-related protein 1, a mediator of mitochondrial fission, in apoptosis. Dev Cell 2001; 1: 515-525.

28. Gandre-Babbe S, van der Bliek AM. The novel tail-anchored membrane protein Mff controls mitochondrial and peroxisomal fission in mammalian cells. Mol Biol Cell 2008; 19: 2402-2412.

29. Imoto M, Tachibana I, Urrutia R. Identification and functional characterization of a novel human protein highly related to the yeast dynamin-like GTPase Vps1p. J Cell Sci 1998; 111: 1341-1349.

30. Smirnova E, Griparic L, Shurland DL, van der Bliek AM. Dynaminrelated protein Drp1 is required for mitochondrial division in mammalian cells. Mol Biol Cell 2001; 12: 2245-2256.

31. James DI, Parone PA, Mattenberger Y, Martinou JC. hFis1, a novel component of the mammalian mitochondrial fission machinery. $J$ Biol Chem 2003; 278: 36373-36379.

32. Yoon Y, Krueger EW, Oswald BJ, McNiven MA. The mitochondrial protein hFis1 regulates mitochondrial fission in mammalian cells through an interaction with the dynamin-like protein DLP1. Mol Cell Biol 2003; 23: 5409-5420.

33. Varadi A, Johnson-Cadwell LI, Cirulli V, Yoon Y, Allan VJ, Rutter GA. Cytoplasmic dynein regulates the subcellular distribution of mitochondria by controlling the recruitment of the fission factor dynaminrelated protein-1. J Cell Sci 2004; 117: 4389-4400.

34. Benard G, Bellance N, James D, Parrone P, Fernandez H, Letellier $\mathrm{T}$, et al. Mitochondrial bioenergetics and structural network organization. J Cell Sci 2007; 120: 838-848.

35. Shaw JM, Nunnari J. Mitochondrial dynamics and division in budding yeast. Trends Cell Biol 2002; 12: 178-184.

36. Stojanovski D, Koutsopoulos OS, Okamoto K, Ryan MT. Levels of human Fis1 at the mitochondrial outer membrane regulate mitochondrial morphology. J Cell Sci 2004; 117: 1201-1210.

37. Suzuki M, Jeong SY, Karbowski M, Youle RJ, Tjandra N. The solution structure of human mitochondria fission protein Fis1 reveals a novel TPR-like helix bundle. J Mol Biol 2003; 334: 445-458.

38. Lee YJ, Jeong SY, Karbowski M, Smith CL, Youle RJ. Roles of the mammalian mitochondrial fission and fusion mediators Fis1, Drp1, and Opa1 in apoptosis. Mol Biol Cell 2004; 15: 5001-5011.

39. Taylor SW, Fahy E, Zhang B, Glenn GM, Warnock DE, Wiley S, et al. Characterization of the human heart mitochondrial proteome. Nat Biotechnol 2003; 21: 281-286.

40. Karbowski M, Neutzner A, Youle RJ. The mitochondrial E3 ubiquitin ligase MARCH5 is required for Drp1 dependent mitochondrial division. J Cell Biol 2007; 178: 71 -84.

41. Nakamura N, Kimura Y, Tokuda M, Honda S, Hirose S. MARCH-V is a novel mitofusin 2- and Drp1-binding protein able to change mitochondrial morphology. EMBO Rep 2006; 7: 1019-1022.

42. Yonashiro R, Ishido S, Kyo S, Fukuda T, Goto E, Matsuki Y, et al. A novel mitochondrial ubiquitin ligase plays a critical role in mitochondrial dynamics. EMBO J 2006; 25: 3618-3626.

43. Yonashiro R, Sugiura A, Miyachi M, Fukuda T, Matsushita N, Inatome R, et al. Mitochondrial ubiquitin ligase MITOL ubiquitinates mutant SOD1 and attenuates mutant SOD1-induced reactive oxygen species generation. Mol Biol Cell 2009; 20: 4524-4530.

44. Sugiura A, Yonashiro R, Fukuda T, Matsushita N, Nagashima S, Inatome R, et al. A mitochondrial ubiquitin ligase MITOL controls cell toxicity of polyglutamine-expanded protein. Mitochondrion 2011; 11: $139-146$.

45. Chai Y, Koppenhafer SL, Shoesmith SJ, Perez MK, Paulson HL. Evidence for proteasome involvement in polyglutamine disease: Localization to nuclear inclusions in SCA3/MJD and suppression of polyglutamine aggregation in vitro. Hum Mol Genet 1999; 8: $673-$ 682 .

46. Honda HM, Korge P, Weiss JN. Mitochondria and ischemia/reperfusion injury. Ann NY Acad Sci 2005; 1047: 248-258.

47. Li W, Bengtson MH, Ulbrich A, Matsuda A, Reddy VA, Orth A, et al. Genome-wide and functional annotation of human E3 ubiquitin ligases identifies MULAN, a mitochondrial E3 that regulates the organelle's dynamics and signaling. PLoS One 2008; 3: e1487.

48. Neuspiel M, Schauss AC, Braschi E, Zunino R, Rippstein P, Rachubinski RA, et al. Cargo-selected transport from the mitochondria to peroxisomes is mediated by vesicular carriers. Curr Biol 2008; 18: $102-108$

49. Braschi E, Zunino R, McBride HM. MAPL is a new mitochondrial SUMO E3 ligase that regulates mitochondrial fission. EMBO Rep 2009; 10: 748-754.

50. Harder Z, Zunino R, McBride H. Sumo1 conjugates mitochondrial substrates and participates in mitochondrial fission. Curr Biol 2004; 14: $340-345$.

51. Zunino R, Schauss A, Rippstein P, Andrade-Navarro M, McBride HM. The SUMO protease SENP5 is required to maintain mitochondrial morphology and function. J Cell Sci 2007; 120: 1178-1188.

52. Zunino R, Braschi E, Xu L, McBride HM. Translocation of SenP5 from the nucleoli to the mitochondria modulates DRP1-dependent fission during mitosis. J Biol Chem 2009; 284: 17783-17795.

53. Hom J, Yu T, Yoon Y, Porter G, Sheu SS. Regulation of mitochondrial fission by intracellular $\mathrm{Ca}^{2+}$ in rat ventricular myocytes. Biochim Biophys Acta 2010; 1797: 913-921.

54. Parra V, Eisner V, Chiong M, Criollo A, Moraga F, Garcia A, et al. Changes in mitochondrial dynamics during ceramide-induced cardiomyocyte early apoptosis. Cardiovasc Res 2008; 77: 387-397.

55. Javadov S, Rajapurohitam V, Kilic A, Hunter JC, Zeidan A, Said Faruq $\mathrm{N}$, et al. Expression of mitochondrial fusion-fission proteins during post-infarction remodeling: The effect of NHE-1 inhibition. Basic Res Cardiol 2011; 106: 99-109.

56. Oka T, Komuro I. Molecular mechanisms underlying the transition of cardiac hypertrophy to heart failure. Circ J 2008; 72(Suppl A): 
A-13-A-16.

57. Palaniyandi SS, Qi X, Yogalingam G, Ferreira JC, Mochly-Rosen D. Regulation of mitochondrial processes: A target for heart failure. Drug Discovery Today Dis Mech 2010; 7: e95-e102.

58. Chen L, Gong Q, Stice JP, Knowlton AA. Mitochondrial OPA1, apoptosis, and heart failure. Cardiovasc Res 2009; 84: 91 -99.

59. Bugger H, Schwarzer M, Chen D, Schrepper A, Amorim PA, Schoepe $\mathrm{M}$, et al. Proteomic remodelling of mitochondrial oxidative pathways in pressure overload-induced heart failure. Cardiovasc Res 2010; 85: 376-384.

60. Kimura A. Molecular etiology and pathogenesis of hereditary cardiomyopathy. Circ J 2008; 72(Suppl A): A-38-A-48.

61. Ausma J, Thone F, Dispersyn GD, Flameng W, Vanoverschelde JL, Ramaekers FC, et al. Dedifferentiated cardiomyocytes from chronic hibernating myocardium are ischemia-tolerant. Mol Cell Biochem 1998; 186: $159-168$

62. Jones M, Ferrans VJ, Morrow AG, Roberts WC. Ultrastructure of crista supraventricularis muscle in patients with congenital heart diseases associated with right ventricular outflow tract obstruction. Circulation 1975; 51: 39-67.

63. Schaper J, Froede R, Hein S, Buck A, Hashizume H, Speiser B, et al. Impairment of the myocardial ultrastructure and changes of the cytoskeleton in dilated cardiomyopathy. Circulation 1991; 83: 504-514.

64. Scholz D, Diener W, Schaper J. Altered nucleus/cytoplasm relationship and degenerative structural changes in human dilated cardiomyopathy. Cardioscience 1994; 5: 127-138.

65. Ashrafian H, Docherty L, Leo V, Towlson C, Neilan M, Steeples V, et al. A mutation in the mitochondrial fission gene Dnm1l leads to cardiomyopathy. PLoS Genet 2010; 6: e1001000.

66. Ago T, Kuroda J, Kamouchi M, Sadoshima J, Kitazono T. Pathophysiological roles of NADPH oxidase/nox family proteins in the vascular system. Circ J 2011; 75: 1791-1800.

67. Ong SB, Subrayan S, Lim SY, Yellon DM, Davidson SM, Hausenloy DJ. Inhibiting mitochondrial fission protects the heart against ischemia/reperfusion injury. Circulation 2010; 121: 2012-2022.
68. Wang JX, Jiao JQ, Li Q, Long B, Wang K, Liu JP, et al. miR-499 regulates mitochondrial dynamics by targeting calcineurin and dynamin-related protein-1. Nat Med 2011; 17: 71-78.

69. Legros F, Lombes A, Frachon P, Rojo M. Mitochondrial fusion in human cells is efficient, requires the inner membrane potential, and is mediated by mitofusins. Mol Biol Cell 2002; 13: 4343-4354.

70. Olichon A, Emorine LJ, Descoins E, Pelloquin L, Brichese L, Gas $\mathrm{N}$, et al. The human dynamin-related protein OPA1 is anchored to the mitochondrial inner membrane facing the inter-membrane space. FEBS Lett 2002; 523: $171-176$.

71. Bossy-Wetzel E, Barsoum MJ, Godzik A, Schwarzenbacher R, Lipton SA. Mitochondrial fission in apoptosis, neurodegeneration and aging. Curr Opin Cell Biol 2003; 15: 706-716.

72. Schwartz AL, Ciechanover A. Targeting proteins for destruction by the ubiquitin system: Implications for human pathobiology. Аnпи Rev Pharmacol Toxicol 2009; 49: 73-96.

73. Johnson ES, Schwienhorst I, Dohmen RJ, Blobel G. The ubiquitinlike protein Smt3p is activated for conjugation to other proteins by an Aos1p/Uba2p heterodimer. EMBO J 1997; 16: 5509-5519.

74. Johnson ES. Protein modification by SUMO. Апnи Rev Biochem 2004; 73: 355-382.

75. Melchior F, Schergaut M, Pichler A. SUMO: Ligases, isopeptidases and nuclear pores. Trends Biochem Sci 2003; 28: 612-618.

76. Tatham MH, Jaffray E, Vaughan OA, Desterro JM, Botting CH, Naismith $\mathrm{JH}$, et al. Polymeric chains of SUMO-2 and SUMO-3 are conjugated to protein substrates by SAE1/SAE2 and Ubc9. J Biol Chem 2001; 276: 35368-35374.

77. Bossis G, Melchior F. SUMO: Regulating the regulator. Cell Div 2006; 1: 13.

78. Marx J. Cell biology: SUMO wrestles its way to prominence in the cell. Science 2005; 307: 836-839.

79. Saitoh H, Hinchey J. Functional heterogeneity of small ubiquitinrelated protein modifiers SUMO-1 versus SUMO-2/3. J Biol Chem 2000; 275: 6252-6258. 